\title{
Random access transport capacity of multihop AF relaying: a throughput-reliability tradeoff
}

\author{
Jaeyoung Lee ${ }^{1}$, Sung-il Kim¹, Saejoon $\mathrm{Kim}^{2}$ and Jun Heo ${ }^{1 *}$
}

\begin{abstract}
To determine the capacity of distributed wireless networks (i.e., ad hoc networks), the random access transport capacity was proposed as the average maximum rate of successful end-to-end transmission in the distance. In this article, we consider the random access transport capacity for multihop relaying to find the end-to-end throughput of a wireless ad hoc network, where each node relays the signal using an amplify-and-forward (AF) strategy. In particular, we analyze the exact outage probability for multihop AF relaying in the presence of both co-channel interference and thermal noise, where interferers are spatially distributed following a Poisson distribution. In our numerical results, it is observed that the maximum random access transport capacity is achieved at a specific spatial density of transmitting nodes due to the throughput-reliability tradeoff as the number of transmitting nodes (=interferers) increases. We compute the optimal spatial density of transmitting nodes that maximize their random access transport capacity. As a result, we can obtain the actual random access transport capacity of multihop AF relaying and predict the maximum number of transmitting nodes per unit area to maximize their performance.
\end{abstract}

Keywords: Amplify-and-forward (AF), Multihop relaying, Interference, Random access transport capacity, Poisson network, Throughput-reliability tradeoff

\section{Introduction}

Cooperative communication is a promising and emerging technique for enhancing the coverage and reliability in wireless networks $[1,2]$. In particular, dual-hop transmission systems employing amplify-and-forward (AF) relaying, where a relay simply retransmits a scaled version of the received signal to the destination, are being spotlighted, due to their low complexity and delay benefits. In addition, the performance analysis of dual-hop AF systems has been an important area of research in recent years [3-5]. However, since dual-hop transmission over long distances requires a very high transmission power, multihop transmission in which a source communicates with a destination via a number of relays has been proposed as an effective method of establishing connectivity between the nodes of a network [6,7]. More recently, the

\footnotetext{
${ }^{*}$ Correspondence: junheo@korea.ac.kr

${ }^{1}$ The School of Electrical Engineering, Korea University, 5-1 Anam-dong,

Sungbuk-gu,Seoul, 136-713, Republic of Korea

Full list of author information is available at the end of the article
}

multihop transmission with AF strategy has drawn considerable attention in the literature [8-10]. In [8,9] examined the ergodic capacity and outage probability of multihop transmission with AF strategy using Jensen' inequality and the inequality between the harmonic and geometric means. [10] computed the optimal number of hops for linear multihop AF relaying with equal resource allocation in terms of the random coding error exponent. All of these previous works on multihop AF relaying focused on noiselimited fading environments for ideal configurations without interference. Since network interference is inevitable in practical wireless networks, due to spectral reuse, AF relaying in the presence of co-channel interference has been studied [11-14]. However, [11,12] considered only dual-hop transmission and [11-13] neglected either noise or interference at each node for analytical tractability. Furthermore, most of the prior works on AF relay networks, including [14], analyzed the outage probability using some approximation methods, such as the harmonic mean, and assumed that the locations of the network nodes are deterministic without spatial randomness.

\section{量 Springer}

(c) 2013 Lee et al.; licensee Springer. This is an Open Access article distributed under the terms of the Creative Commons Attribution License (http://creativecommons.org/licenses/by/2.0), which permits unrestricted use, distribution, and reproduction in any medium, provided the original work is properly cited. 
Meanwhile, under the assumption that the interferer locations are random, there have been some state-ofthe-art works on the ad hoc network capacity [15-23]. In [15], the transmission capacity was proposed as the maximum allowable density of transmitting nodes to satisfy the data rate and outage probability constraints. The transmission capacity framework has been successfully used to characterize the physical layer on the ad hoc networks [15-20], such as through the use of multiple antennas [17], interference cancellation [18] and spectrum sharing in tiered cellular networks [20]. [21] further studied the tradeoffs of the transmission capacity between the throughput, delay, and reliability in wireless ad hoc networks. However, since most of the prior works on computing the transmission capacity were limited to single hop transmission without noise, [22] derived the transmission capacity of dual-hop relaying while considering the thermal noise. Recently, to account for multiple hops and retransmissions, [23] developed a new metric for quantifying the end-to-end throughput termed random access transport capacity. The random access transport capacity was defined as the average maximum rate of successful end-to-end transmissions over some distance.

In this article, we consider a realistic communication environment in wireless ad hoc networks where all of the transmitting nodes are randomly scattered and uncoordinated following a Poisson law over a plane. For decentralized wireless networks, we consider the random access transport capacity to find the overall end-to-end throughput for multihop AF relaying. To compute the random access transport capacity, we analyze the exact outage probability of multihop transmission with AF strategy in a Poisson field of interferers without neglecting the noise at all of the nodes. From our numerical results, we observe that even when the number of transmitting nodes increases, the overall throughput of multihop relaying does not increase consistently due to interference. In other words, the random access transport capacity has a maximum value at a certain spatial density of transmitting nodes, due to the throughput-reliability tradeoff, and shows that the increase in the throughput is inversely proportional to the reliability as the number of transmitting nodes(=interferers) increases. Thus, in this article, we compute the optimal spatial density of transmitting nodes that maximize their random access transport capacity, and this helps us to predict and manage the maximum available number of transmitting nodes per unit area to maximize their performance. Moreover, since each relay node amplifies the interference, as well as the thermal noise, our results show that the performance of multihop AF relaying can be degraded due to the accumulated interference caused by the increasing number of transmission hops.
The remainder of this article is organized as follows. Section 2 presents the system model with channel model and interference model. We analyze the random access transport capacity of multi-hop AF relaying with both noise and Poisson interference in Section 3. Section 4 presents the optimal density of transmitting nodes to maximize their random access transport capacity. Section 5 compares the simulation results with the analytical results. Finally, Section 6 concludes this article.

\section{System model}

We consider a large wireless ad hoc network where source node $S$ drawn from a homogeneous poisson point process (PPP) on a plane of intensity $\lambda$ wishes to communicate with a destination node $D$ that is at a distance $d_{S D}$ away in a random direction. Each source node communicates with its assigned destination node via intermediate relay nodes $R$ which transmit their received data using an AF strategy to their respective successor nodes by means of a process called linear multihop relaying, as illustrated in Figure 1. Thus, as all transmitters (sources or relays) in each hop communicate with their assigned receivers (relays or destinations) in a random direction, all of the transmitting nodes are randomly distributed following a Poisson distribution with spatial density $\lambda$ due to the random translation invariance property of PPP [24]. In this article, as it is difficult to compute the overall throughput in a general model for ad hoc networks, three assumptions are made to make the calculation more tractable. First, we assume that each source transmits a single packet at a time slot along the entire multihop path: no intraroute spatial reuse. Second, all of the nodes in the $n$th hop transmit data to their own assigned receivers using the same power $P_{n}$. Finally, we assume that all of pairs of transmitters-receivers in the $n$th hop are the same distance apart $d_{n}\left(\sum_{n=1}^{K} d_{n}=d_{S D}\right)$. This is obviously a simplification, but it constitutes an important special case of general ad hoc networks, and these assumptions allow a closed-form for the end-to-end throughput in terms of the key network parameters. In this article, all of the nodes are assumed to have a single antenna operating in a common frequency band and to be in half-duplex mode.

\subsection{Channel model}

We consider a channel model with small scale fading and large scale path loss for a practical scenario. Therefore, the square of the instantaneous channel response between any pair of nodes located at $x_{1}$ and $x_{2}$ can be rewritten as

$$
\left|h_{12}\right|^{2}=F_{12} \cdot l\left(\left\|x_{1}-x_{2}\right\|\right),
$$

where $F_{12}$ captures the small scale fading which obeys a Rayleigh fading model and $l\left(\left\|x_{1}-x_{2}\right\|\right)=\left\|x_{1}-x_{2}\right\|^{-\alpha}$ characterizes the effect of large scale path loss with path 


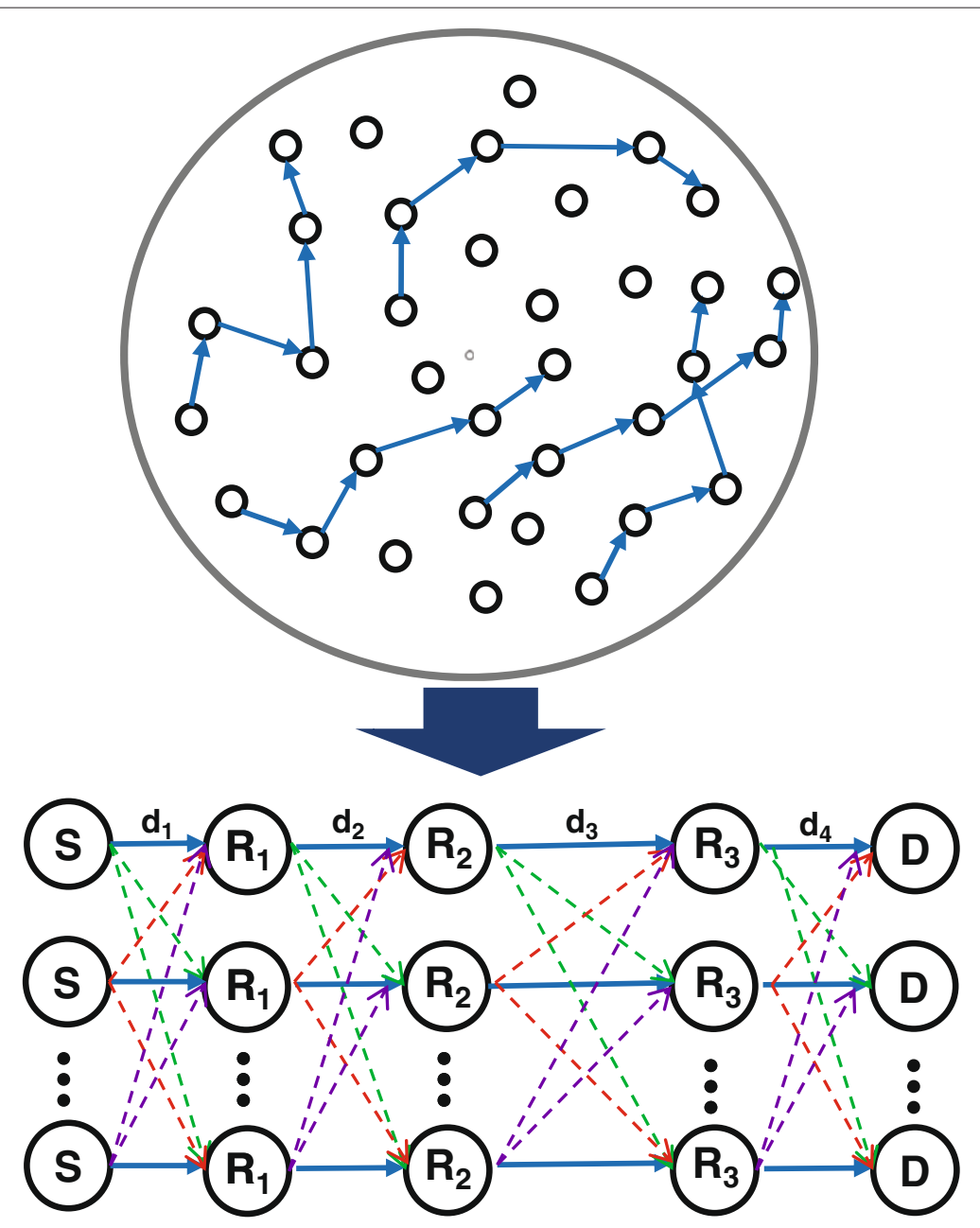

Figure 1 A multihop relay network consisting of source, relays, and destination in a wireless ad hoc networks. The sets of nodes can be divided into multiple different pairs for each transmission hop. The dashed lines represent interference which is generated by sources and relays in each transmission hop.

loss exponent $\alpha$. In this article, we consider $2<\alpha<$ 5 for the main physical range of interest for path loss exponent in wireless networks. We assume that the channel responses for all of the nodes are independent and quasi-static.

\subsection{Interference model}

We consider a slotted ALOHA transmission without centralized scheduling $[19,22,23]$, where all of the transmitters which cause interference are randomly scattered and uncoordinated. Since all of nodes are drawn from a homogeneous 2-D PPP of intensity $\lambda$, all nodes generating interference at the $n$th hop transmission are also modeled as a homogeneous PPP with $\lambda$. Then, the sets of interferers in each hop are denoted as $\mathcal{I}:=\left\{I x_{n}, n \in \mathbb{N}\right\}$. In particular, we define the interferer set as $\Phi_{I_{n}}:=\left\{I_{n}\right\}$ where $I_{n}$ is the location of interferers. In the case of the transmission power, we assume that all of the interferers in the $n$th hop use the same power $P_{I_{n}}$. Moreover, as the only $\alpha>2$ for which the aggregate interference has a distribution expressible in closed-form is for $\alpha=4[19,25]$, we assume a large wireless ad hoc network where each multihop branch is located relatively far away with each other.

\section{Random access transport capacity of multihop AF relaying}

In this section, we consider the random access transport capacity, defined as the average maximum rate of successful end-to-end transmission over some distance. Unlike the conventional capacities of wireless ad hoc networks, the random access transport capacity is a suitable metric to measure the overall throughput of the transmitting nodes in a decentralized wireless network. For computing the random access transport capacity of wireless ad hoc networks, while [23] considered the multihop 
transmission in the presence of retransmissions, we focus on the mutltihop AF relaying the most popular protocol due to low complexity and no retransmission. The definition of the random access transport capacity is

$$
C=\lambda d \log (1+\zeta) p_{s} \quad(\mathrm{bps} / \mathrm{Hz} / \mathrm{m})
$$

where $p_{s}$ is the probability that the packet is successfully decoded by the destination $D, \lambda$ is the density of transmitting nodes (=interferers), $\zeta$ is the target SINR, and $d$ is end-to-end distance. To compute the success probability of the random access transport capacity, the outage probability of multi-hop AF relaying should be analyzed first.

In the first transmission hop, the relay nodes receive the signal transmitted from the source nodes and the received signal $y_{1}$ at the relay can be expressed as

$$
y_{1}=h_{1} s_{0}+\sum_{i \in \Phi_{I_{1}}} l_{i} w_{i}+n_{1}
$$

where $s_{0}$ is the transmitted symbol with $E\left[\left|s_{0}\right|^{2}\right]=P_{1}$, $h_{1}$ is the channel of the first transmission hop, and $n_{1}$ is the additive white Gaussian noise with an average power of $\sigma_{1}^{2}$. In this article, we regard the aggregate of interference in Poisson field $\sum_{i \in \Phi_{I_{1}}} l_{i} w_{i}$ as a shot noise [15-23,26] where the relay node $R_{1}$ is affected by its interferers located in a homogenous PPP $\Phi_{I_{1}}$ and $w_{i}$ denotes the transmitted symbol of interferer $i$. In addition, the average transmit power of an interferer is $E\left[\left|s_{i}\right|^{2}\right]=P_{I_{1}}$ and $\left\{l_{i}\right\}_{i \in \Phi_{S}}$ are the channels from the interferer $i$ to the first relay node $R_{1}$. Using the AF strategy, the received signal at the second hop can be written as

$$
y_{2}=h_{2} G_{2} y_{1}+\sum_{j \in \Phi_{I_{2}}} l_{j} w_{j}+n_{2}
$$

where $h_{2}$ is the channel of the second transmission hop and $n_{2}$ is the additive white Gaussian noise with an average power of $\sigma_{2}^{2} .\left\{l_{j}\right\}_{j \in \Phi_{I_{2}}}$ are the channels from the interferer $j$ to the respective successor node and the average transmit power of an interferer in the second hop is $E\left[\left|w_{j}\right|^{2}\right]=P_{I_{2}}$. In the AF strategy, as the amplification process consists of a simple normalization of the total received power without further processing in the analogue domain, the amplification factor $G_{2}$ of AF relaying with interference [11] can be expressed as

$$
G_{2}=\sqrt{\frac{P_{2}}{\left\|y_{1}\right\|^{2}}}=\sqrt{\frac{P_{2}}{P_{1}\left|h_{1}\right|^{2}+\sum_{i \in \Phi_{I_{1}}}\left|l_{i}\right|^{2} P_{I_{1}}+\sigma_{1}^{2}}} .
$$

From the above results, the received signal $y_{n}$ at the $n$th hop is given by

$$
y_{n}=h_{n} G_{n} y_{n-1}+\sum_{j \in \Phi_{I_{n}}} l_{j} w_{j}+n_{n}, \text { for } n=2, \ldots, K
$$

where $G_{n}=\sqrt{\frac{P_{n}}{\left\|y_{n-1}\right\|^{2}}}$.

Theorem 1 (End-to-end SINR). The end-to-end SINR of multihop AF relaying in the presence of both noise and interference can be written as

$$
\gamma_{e q}=\frac{1}{\prod_{n=1}^{K}\left(1+\frac{1}{\gamma_{n}}\right)-1}
$$

where the SINR of each hop $\gamma_{n}$ is given by

$$
\gamma_{n}=\frac{\left|h_{n}\right|^{2} P_{n}}{\sum_{i \in \Phi_{I_{n}}}\left|g_{i}\right|^{2} P_{I_{n}}+\sigma_{n}^{2}}, \quad \text { for } n=1,2, \ldots, K .
$$

Proof 1. See Appendix 1.

Based on the above end-to-end SINR, the overall achievable rate $R_{\mathrm{e} q}$ can be written as

$$
R_{\mathrm{e} q}=\beta_{n} \log _{2}\left(1+\gamma_{\mathrm{e} q}\right)
$$

where $\beta_{n}$ is the fraction of time for the $n$th hop and is allocated equally with $\beta_{n}=\frac{1}{K}$ in this article. Then, the outage probability of multihop AF relaying can be written as

$P_{\text {out }}(R)=\mathbb{P}\left\{\frac{1}{K} \log _{2}\left(1+\gamma_{\mathrm{e} q}\right)<R\right\}=\mathbb{P}\left\{\gamma_{\mathrm{e} q}<2^{K R}-1\right\}$

where $R$ is the spectral efficiency. From the outage probability, we can also obtain the target $\operatorname{SINR}, \zeta=2^{K R}-1$, in the random access transport capacity. To compute the outage probability of multihop AF relaying, since the endto-end SINR is composed of each single transmission hop, the probability density function $(P D F)$ and cumulative density function $(C D F)$ of each hop [22] can be obtained as

$$
\begin{aligned}
& F_{\gamma_{n}}(x)=1-\exp \left[-\left(\frac{d_{n}^{\alpha} \sigma_{n}^{2}}{P_{n}} x+\frac{\pi^{2} \operatorname{erf}(\sqrt{r}) \lambda}{2} \sqrt{\left.\frac{d_{n}^{\alpha} P_{I_{n}}}{P_{n}} x\right)}\right),\right. \\
& p_{\gamma_{n}}(x)=\left(\frac{d_{n}^{\alpha} \sigma_{n}^{2}}{P_{n}}+\frac{\pi^{2} \operatorname{erf}(\sqrt{r}) \lambda \sqrt{d_{n}^{\alpha} P_{I_{n}}}}{4 \sqrt{P_{n} x}}\right) \\
& \exp \left[-\left(\frac{d_{n}^{\alpha} \sigma_{n}^{2}}{P_{n}} x+\frac{\pi^{2} \operatorname{erf}(\sqrt{r}) \lambda}{2} \sqrt{\frac{d_{n}^{\alpha} P_{I_{n}}}{P_{n}} x}\right)\right], x \geq 0
\end{aligned}
$$

where $d_{n}$ is the distance of each hop, the error function is $\operatorname{erf}(x) \triangleq \frac{2}{\sqrt{\pi}} \int_{0}^{x} \mathrm{e}^{-t^{2}} \mathrm{~d} t$, and $r$ denotes the radius of the 
interference area. Let $\left(1+\frac{1}{\gamma_{n}}\right)$ be $\mathrm{X}_{n}$ and the $P D F$ of $\mathrm{X}_{n}$ is given by

$$
\begin{aligned}
p_{\mathrm{X}_{n}}(x)=\frac{1}{(x-1)^{2}}\left(\frac{d_{n}^{\alpha} \sigma_{n}^{2}}{P_{n}}+\frac{\pi^{2} \operatorname{erf}(\sqrt{r}) \lambda \sqrt{d_{n}^{\alpha} P_{I_{n}}(x-1)}}{4 \sqrt{P_{n}}}\right) \\
\times \exp \left[-\left(\frac{d_{n}^{\alpha} \sigma_{n}^{2}}{P_{n}(x-1)}\right.\right. \\
\quad+\frac{\pi^{2} \operatorname{erf}(\sqrt{r}) \lambda}{2} \sqrt{\left.\left.\frac{d_{n}^{\alpha} P_{I_{n}}}{P_{n}(x-1)}\right)\right], x \geq 1 .}
\end{aligned}
$$

Theorem 2 (Outage probability of multihop AF relaying). The exact outage probability of multihop AF relaying in the presence of both interference and noise can be written as

$$
\begin{aligned}
& P_{\text {out }}(R) \\
& \quad=\int_{0}^{2^{K R-1}} \int_{1}^{1+\frac{1}{s}} \int_{1}^{x_{K-1}} \cdots \int_{1}^{x_{2}} \\
& \frac{1}{s^{2}}\left[\prod_{n=1}^{K-1} \frac{1}{x_{n}} p_{\mathrm{x}_{n}}\left(\frac{x_{n}}{x_{n-1}}\right)\right] p_{\mathrm{X}_{K}}\left(\frac{s+1}{s x_{K-1}}\right) d x_{1} \cdots d x_{K-1} d s
\end{aligned}
$$

where $x_{0}=1$.

Proof 2. See Appendix 2.

In contrast to above result, the conventional outage probability of multihop AF relaying without approximation methods $[13,14]$ can be expressed as

$$
P_{\text {out }}(R)=\int \ldots \iint_{\gamma_{\mathrm{eq}}} p_{\gamma_{1}}\left(x_{1}\right) \cdots \gamma_{n}\left(x_{n}\right) d x_{1} \ldots d x_{n}
$$

where $p_{\gamma_{n}}\left(x_{n}\right)$ is the PDF of $n$th hop. In this case, the complexity of computing the exact outage probability increases as the number of hops increases, because setting the interval of integration for each variable from (7) is very difficult. However, from our result (13), we can obtain the exact outage probability of multihop AF relaying with low complexity by using only the $p \mathrm{x}_{n}(x)$.

Corollary 1 (Special case: Dual-hop AF relaying). The outage probability of dual-hop AF relaying can be written as

$$
\begin{aligned}
P_{\text {out }}(R) & =1-\int_{\varepsilon}^{\infty}\left(B+\frac{D}{2 \sqrt{y}}\right) \\
\exp & {\left[-\left(A\left(\frac{\varepsilon(y+1)}{y-\varepsilon}\right)+B y+C \sqrt{\frac{\varepsilon(y+1)}{y-\varepsilon}}+D \sqrt{y}\right)\right] d y }
\end{aligned}
$$

where

$$
\begin{aligned}
& \varepsilon=2^{2 R}-1, \quad A=\frac{d_{1}^{\alpha} \sigma_{1}^{2}}{P_{1}}, \quad B=\frac{d_{2}^{\alpha} \sigma_{2}^{2}}{P_{2}}, \\
& C=\frac{\pi^{2} \operatorname{erf}(\sqrt{r}) \lambda}{2} \sqrt{\frac{d_{1}^{\alpha} P_{I_{1}}}{P_{1}}}, \quad D=\frac{\pi^{2} \operatorname{erf}(\sqrt{r}) \lambda}{2} \sqrt{\frac{d_{2}^{\alpha} P_{I_{2}}}{P_{2}} .}
\end{aligned}
$$

Proof 3. See [26].

Based on the outage probability, the random access transport capacity of multihop AF relaying $C_{A F}$ can be written as

$$
C_{A F}=\lambda \log (1+\zeta) d_{S D}\left[1-P_{\mathrm{out}}(R)\right] \quad(\mathrm{bps} / \mathrm{Hz} / \mathrm{m})
$$

where $1-P_{\text {out }}(R)$ is the success probability, $\lambda$ is the density of interferers, $\zeta$ is the target $\operatorname{SINR}$ with $2^{K R}-1$, and $d_{S D}$ is the distance between the source and destination. From the above random access transport capacity, we can find the spatial density of successful transmissions at rate $\log (1+\zeta)$ that spans a distance $d_{S D}$ when each node relays its data using the AF strategy.

\section{Optimal spatial density of transmitting nodes for multihop AF relaying}

From numerical results, intriguingly, it is found that the actual random access transport capacity does not increase continuously as the density of transmitting nodes increases, because the interference will increase with increasing number of transmitting nodes. This phenomenon is due to the tradeoff between the throughput and reliability that involves decreasing reliability with increasing throughput as the number of transmitting nodes (=interferers) increases. Moreover, this means that the maximum random access transport capacity is achieved at a specific spatial density of transmitting nodes. Thus, in this section, to maximize their random access transport capacity, we compute the optimal spatial density of transmitting nodes for multihop AF relaying in a wireless ad hoc network. However, since the exact outage probability of multihop AF relaying (13) is very hard to deal with, we approximate the end-to-end SINR in the form of (7), using the following properties which are widely used in the literature $[10,14]$ as

$$
\begin{aligned}
\gamma_{\mathrm{e} q} & =\frac{1}{\prod_{n=1}^{K}\left(1+\frac{1}{\gamma_{n}}\right)-1} \leq \frac{1}{\sum_{n=1}^{K} \frac{1}{\gamma_{n}}} \\
& \leq \min \left(\gamma_{1}, \gamma_{2}, \ldots, \gamma_{K}\right)=\gamma_{\mathrm{e} q}^{\mathrm{u} p}, \quad \text { for all } \gamma_{n} \geq 0 .
\end{aligned}
$$

Since we have already obtained the actual random access transport capacity of multihop AF relaying, this upper bound is used only to compute the optimal spatial density 
of transmitting nodes. Using (18), the outage probability of multihop AF relaying can be written as

$$
\begin{aligned}
P_{\mathrm{out}}(R) & =\mathbb{P}\left\{\frac{1}{K} \log _{2}\left(1+\gamma_{\mathrm{e} q}^{\mathrm{u} p}\right)<R\right\} \\
& =1-\mathbb{P}\left\{\gamma_{1}>2^{K R}-1, \ldots, \gamma_{K}>2^{K R}-1\right\} \\
& =1-\prod_{n=1}^{K}\left[1-F_{\gamma_{n}}\left(2^{K R}-1\right)\right]
\end{aligned}
$$

where the $C D F$ of each single hop transmission $F_{\gamma_{n}}(x)$ was already obtained in (11). Therefore, the approximated outage probability of multihop AF relaying is given by

$$
\begin{gathered}
P_{\text {out }}(R)=1-\exp \left[-\left\{\sum _ { n = 1 } ^ { K } \left(\frac{d_{n}^{\alpha} \sigma_{n}^{2}}{P_{n}}\left(2^{K R}-1\right)+\frac{\pi^{2} \operatorname{erf}(\sqrt{r}) \lambda}{2}\right.\right.\right. \\
\sqrt{\left.\left.\left.\frac{d_{n}^{\alpha} P_{I_{n}}\left(2^{K R}-1\right)}{P_{n}}\right)\right\}\right] .}
\end{gathered}
$$

Based on the above results, the upper bound of the random access transport capacity for multihop AF relaying can be written as

$$
\begin{aligned}
& C_{A F}^{\mathrm{u} p p}=\lambda \log (1+\zeta) d_{S D} \\
& \exp [-\left\{\sum _ { n = 1 } ^ { K } \left(\frac{d_{n}^{\alpha} \sigma_{n}^{2}}{P_{n}}\left(2^{K R}-1\right)\right.\right. \\
&\left.\left.\left.+\frac{\pi^{2} \operatorname{erf}(\sqrt{r}) \lambda}{2} \sqrt{\frac{d_{n}^{\alpha} P_{I_{n}}\left(2^{K R}-1\right)}{P_{n}}}\right)\right\}\right] .
\end{aligned}
$$

Before computing the optimal density of transmitting nodes, we prove that the random access transport capacity is concave in terms of the spatial density $\lambda$. To use the property of concave $\frac{\partial^{2} C_{A F}^{\mathrm{u} p p}}{\partial^{2} \lambda}<0, \frac{\partial^{2} C_{A F}^{\mathrm{upp} p}}{\partial^{2} \lambda}$ can be expressed as

$$
\begin{aligned}
& \frac{\partial^{2} C_{A F}^{\mathrm{u} p p}}{\partial^{2} \lambda}= \log (1+\zeta) d_{S D} \\
& \exp \left[-\left\{\sum _ { n = 1 } ^ { K } \left(\frac{d_{n}^{\alpha} \sigma_{n}^{2}}{P_{n}}\left(2^{K R}-1\right)\right.\right.\right. \\
&+\frac{\pi^{2} \operatorname{erf}(\sqrt{r}) \lambda}{2} \sqrt{\left.\left.\left.\frac{d_{n}^{\alpha} P_{I_{n}}\left(2^{K R}-1\right)}{P_{n}}\right)\right\}\right]} \\
& \times\left(\sum_{n=1}^{K} \frac{\pi^{2} \operatorname{erf}(\sqrt{r})}{2} \sqrt{\left.\frac{d_{n}^{\alpha} P_{I_{n}}\left(2^{K R}-1\right)}{P_{n}}\right)}\right. \\
& \times\left(\sum_{n=1}^{K} \frac{\pi^{2} \operatorname{erf}(\sqrt{r}) \lambda}{2} \sqrt{\frac{d_{n}^{\alpha} P_{I_{n}}\left(2^{K R}-1\right)}{P_{n}}}-2\right) .
\end{aligned}
$$

Then, the random access transport capacity of multihop AF relaying will be concave under the following conditions:

$$
\lambda \leq \frac{4}{\pi^{2} \operatorname{erf}(\sqrt{r})\left(\sum_{n=1}^{K} \sqrt{\frac{d_{n}^{\alpha} P_{I_{n}}\left(2^{K R}-1\right)}{P_{n}}}\right)}=\lambda_{\mathrm{o} p t}^{\max } .
$$

On the contrary, in the region of $\lambda>\lambda_{\mathrm{o} p t}^{\max }$, the random access transport capacity is a monotonic decreasing function due to $\frac{\partial^{3} C_{A F}^{\mathrm{upp}}}{\partial^{3} \lambda}<0$. Thus, the optimal spatial density of transmitting nodes exists in the region of $\lambda \leq \lambda_{\mathrm{o} p t}^{\max }$.

Using the upper bound for multihop AF relaying, the optimal density of transmitting nodes $\lambda_{\text {opt }}^{\text {upp which maxi- }}$ mizes the random access transport capacity can be written as

$$
\lambda_{\mathrm{o} p t}^{\mathrm{u} p p}=\arg \max _{\lambda} C_{A F}^{\mathrm{u} p p} .
$$

Applying (21), the optimization problem after eliminating the constant can be rewritten as

$$
\begin{aligned}
\lambda_{\text {opt }}^{\text {upp }} & =\arg \max _{\lambda} \lambda \exp \left[-\left\{\sum _ { n = 1 } ^ { K } \left(\frac{\pi^{2} \operatorname{erf}(\sqrt{r}) \lambda}{2}\right.\right.\right. \\
& \left.\left.\left.\sqrt{\left.\frac{d_{n}^{\alpha} P_{I_{n}}\left(2^{K R}-1\right)}{P_{n}}\right)}\right)\right\}\right] \\
& =\arg \min _{\lambda} f(\lambda)
\end{aligned}
$$

where

$$
f(\lambda)=\sum_{n=1}^{K}\left(\frac{\pi^{2} \operatorname{erf}(\sqrt{r}) \lambda}{2} \sqrt{\frac{d_{n}^{\alpha} P_{I_{n}}\left(2^{K R}-1\right)}{P_{n}}}\right)-\ln \lambda .
$$

In conclusion, we can obtain the optimal density of trans-

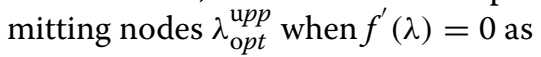

$$
\lambda_{\mathrm{opt}}^{\mathrm{u} p p}=\frac{2}{\pi^{2} \operatorname{erf}(\sqrt{r}) \sqrt{\left(2^{K R}-1\right)}}\left(\frac{1}{\sum_{n=1}^{K} \sqrt{\frac{d_{n}^{\alpha} P_{I_{n}}}{P_{n}}}}\right) .
$$

Using this bound (27), we can predict and manage the maximum number of nodes per unit area that maximizes their random access transport capacity of multihop AF relaying.

\section{Numerical results}

In this section, we present some numerical results concerning the outage probability and random access transport capacity for multihop AF relaying with both interference and noise in a Poisson network. To illustrate our analytic and simulated results, we consider a Rayleigh fading channel having equal resource allocation 
with equal transmission power $P_{n}=P_{T}$, equidistance $d_{n}=d_{S D} / K$, identical interference power $P_{I_{n}}=P_{I N F}$, and fixed noise variance $\sigma_{n}^{2}=1$. Moreover, we consider 30 randomly distributed nodes following a Poisson distribution for each hop with the radius of the interference region $r=\sqrt{\frac{30}{\pi \lambda}}$, where $\lambda$ is the density of transmitting nodes (=interferers).

\subsection{Outage probability}

Figure 2 shows the analytic and simulated outage probability as a function of the transmission power of each hop $P_{T}$ for multihop AF relaying with $K=2$ and 3 considering both noise and interference in a Poisson network. This figure represents the outage probability with end-toend spectral efficiency $R=0.5 \mathrm{bps} / \mathrm{Hz}$ for two different distances between source and destination, $d_{S D}=3$ and 5 , with spatial density of interferers $\lambda=0.01$ and path loss exponent $\alpha=4$. In addition, we assume that the interference power also increases at a rate of 0.05 as the transmitting power increases, where $P_{I N F}=P_{T} \times 0.05$. From this figure, multihop AF relaying with $K=3$ has better outage performance than dual-hop relaying. Likewise, in a relay network without interference, this figure reveals that the outage probability of multihop AF relaying decreases rapidly as the transmission power increases. However, in the high-transmission power regime, the rate of a decline slows and an error floor phenomenon occurs, due to the increasing interference effect on the multihop transmission with increasing interference power. As the interference signals are included in the received signal,

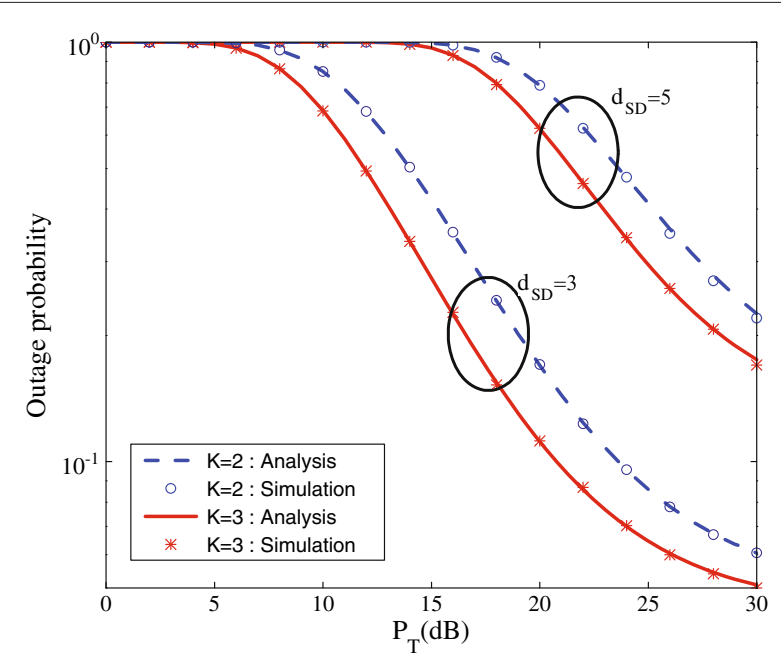

Figure 2 Outage probability of multihop AF relaying with $K=2$ and 3 as a function of $P_{T}$ for different distance between source and destination intensity of interferers $d_{S D}=3$ and 5 at the end-to-end spectral efficiency $R=0.5 \mathrm{bps} / \mathrm{Hz}$ in Rayleigh fading channel with path loss exponent $\alpha=4$, interference power $P_{I N F}=0.05 \times P_{T}$, and density of transmitting nodes $\lambda=0.01$. it is noted that the AF strategy amplifies the interference as well as thermal noise. In addition, we can see from Figure 2 that this analysis agrees exactly with the simulation results.

\subsection{Random access transport capacity}

To identify the throughput-reliability tradeoff, Figures 3 and 4 represent the actual random access transport capacity of multihop AF relaying in a Rayleigh fading channel with distance between source and destination $d_{S D}=5$ at an end-to-end spectral efficiency $R=0.5 \mathrm{bps} / \mathrm{Hz}$. In Figure 3, we plot the random access transport capacity of multihop AF relaying with $K=3$ as a function of the interference power $P_{I N F}$ for different densities of interferers, $\lambda=0.05,0.1$, and 0.2 with transmission power $P_{T}=$ $20 \mathrm{~dB}$ and path loss exponent $\alpha=3$. Obviously, the random access transport capacity decreases continuously as the interference power increases. Interestingly, although some interference is present, the region of $P_{I N F}<5 \mathrm{~dB}$ (solid circle) indicates that multihop AF relaying with a relatively high spatial density, $\lambda=0.1$ and 0.2 , has a higher overall throughput than that with $\lambda=0.05$, because the effect of interference on the multihop relaying is insignificant in the low interference power regime. However, the multihop relaying with the lowest density $\lambda=0.05$ has the largest random access transport capacity in the high interference power regime.

From a different viewpoint, Figure 4. presents the random access transport capacity of multihop AF relaying with $K=2$ and 4 as a function of the spatial density of

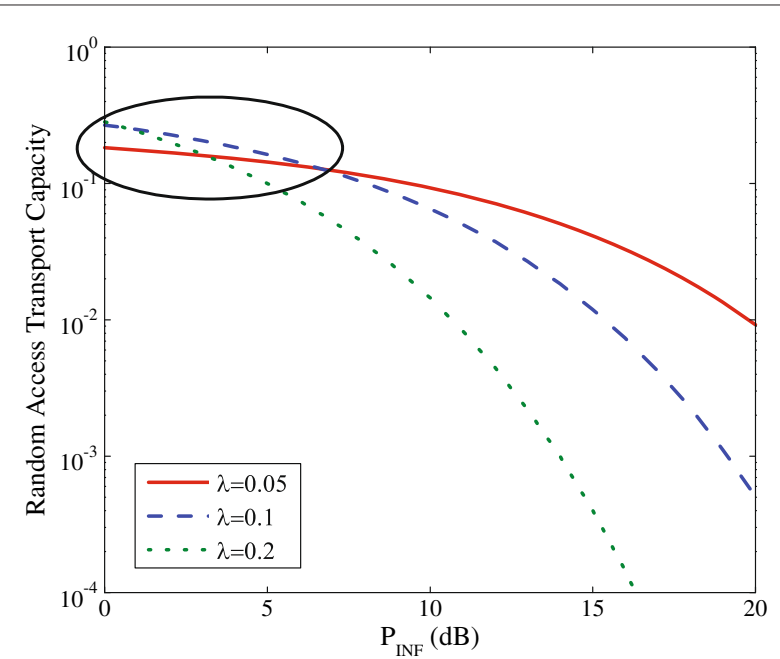

Figure 3 Random access transport capacity of multihop AF relaying with $K=3$ as a function of interference power $P_{I N F}$ for different spatial density $\lambda=0.05,0.1$, and 0.2 at the end-to-end spectral efficiency $R=0.5 \mathrm{bps} / \mathrm{Hz}$ in Rayleigh fading channel with path loss exponent $\alpha=\mathbf{3}$ when transmission power $P_{T}=20 \mathrm{~dB}$, and distance $d_{S D}=\mathbf{5}$. 


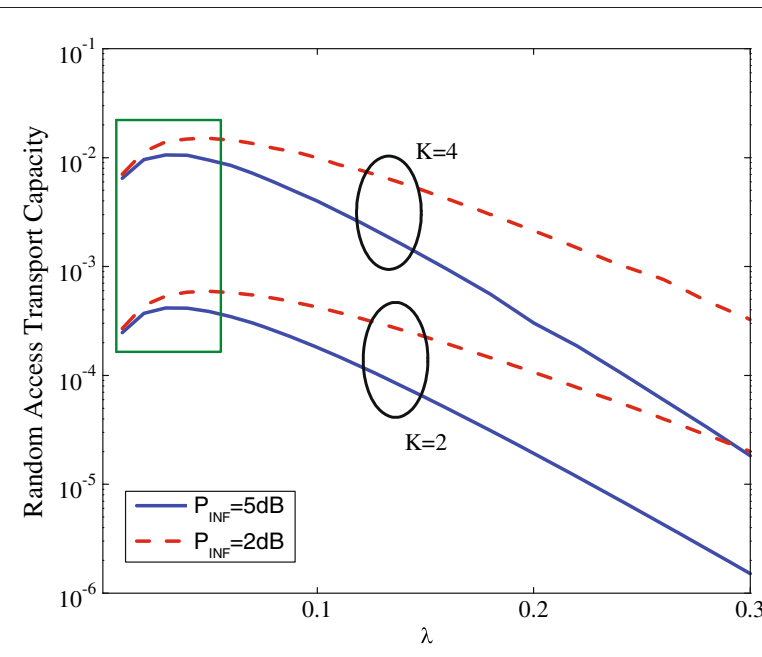

Figure 4 Random access transport capacity of multihop AF relaying as a function of spatial density $\lambda$ for different number of hops $K=\mathbf{2}$ and $\mathbf{4}$ at the end-to-end spectral efficiency $R=0.5 \mathrm{bps} / \mathrm{Hz}$ in Rayleigh fading channel with path loss exponent $\alpha=3$ when transmitting power $P_{T}=15 \mathrm{~dB}$ and distance between source and destination $d_{S D}=5$.

transmitting nodes $\lambda$ for two different interference powers $P_{I N F}=2 \mathrm{~dB}$ and $5 \mathrm{~dB}$ with the fixed transmission power $P_{T}=15 \mathrm{~dB}$. This figure also shows that multihop AF relaying with $K=4$ can achieve higher end-to-end throughput than dual-hop relaying. Meanwhile, the general overall throughput of ad hoc networks increases when many nodes transmit data simultaneously, but the random access transport capacity does not increase consistently owing to the increasing interference as the spatial density of transmitting nodes increases. Especially, in Figure 4, the random access transport capacity has the maximum value at the certain spatial density, because the effect of increasing throughput is larger than that of decreasing reliability in the relatively low-spatial density regime. In both figures, we observe that there is a tradeoff between the throughput and reliability and a need to compute the optimal density of transmitting nodes for the purpose of maximizing the random access transport capacity.

\subsection{Optimal spatial density of transmitting nodes}

For the numerical results, since we assume equal resource allocation $P_{n}=P_{T}, d_{n}=d_{S D} / K$, and $P_{I_{n}}=P_{I N F}$, the upper bound for the optimal density of transmitting nodes (27) can be simplified as follows:

$$
\lambda_{\mathrm{opt}}^{\mathrm{u} p p e r}=\frac{2 \sqrt{P_{T}}}{\pi^{2} \operatorname{erf}(\sqrt{r}) K^{1-\frac{\alpha}{2}} \sqrt{d_{S D}^{\alpha} P_{I N F}\left(2^{K R}-1\right)}} .
$$

Using the above results, to verify the tightness of our upper bound in terms of the optimal density of transmitting nodes, the actual random access transport capacity and its upper bound for multihop AF relaying with $K=3$ are plotted as a function of the spatial density $\lambda$ for different transmission powers $P_{T}$ at an end-to-end spectral efficiency $R=0.5$ in Figure 5 . As the maximum random access transport capacity is considered, we plot it in the relatively low spatial density regime $\lambda \leq 0.2$ when the distance $d_{S D}=4$ and the interference power $P_{I N F}=3 \mathrm{~dB}$. Although there is a big difference between the actual and upper bound values, both values have a maximum random access transport capacity at a similar spatial density. It is because our upper bound is derived to obtain the optimal spatial density of transmitting nodes. For example, the gap between the exact optimal density of transmitting nodes and its upper bound decreases from 0.06 to 0.05 as the transmission power decreases. Since it is very difficult to make closed form using another approximation method based on the PDF and CDF (11), we cannot find more tight bound to satisfy both optimal spatial density and actual random access transport capacity in this article yet. However, from this figure, we note that the optimal spatial density of nodes to maximize their performance can be predicted easily by using our upper bound.

Now, focusing on the upper bound, Figure 6 shows the optimal density of transmitting nodes as a function of the distance between the source and destination $d_{S D}$ for different path loss exponents $\alpha$ and spectral efficiencies $R$, when the transmission power $P_{T}=15 \mathrm{~dB}$ and $P_{I N F}=5 \mathrm{~dB}$. As the end-to-end distance $d_{S D}$ increases, this figure reveals that not only the random

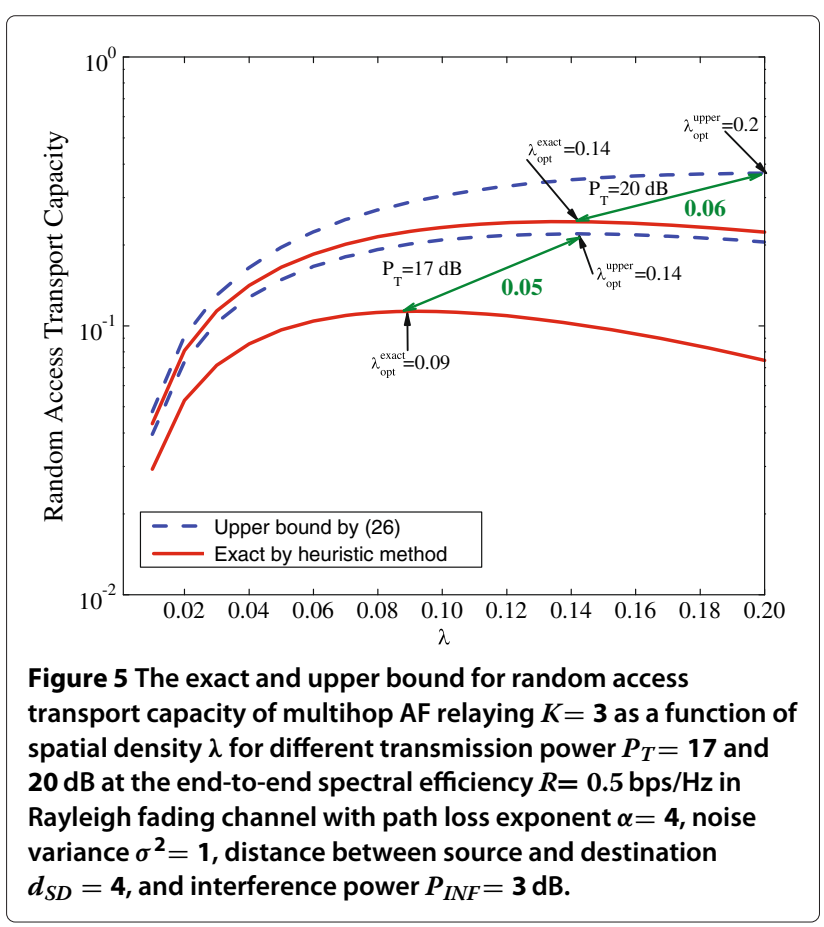




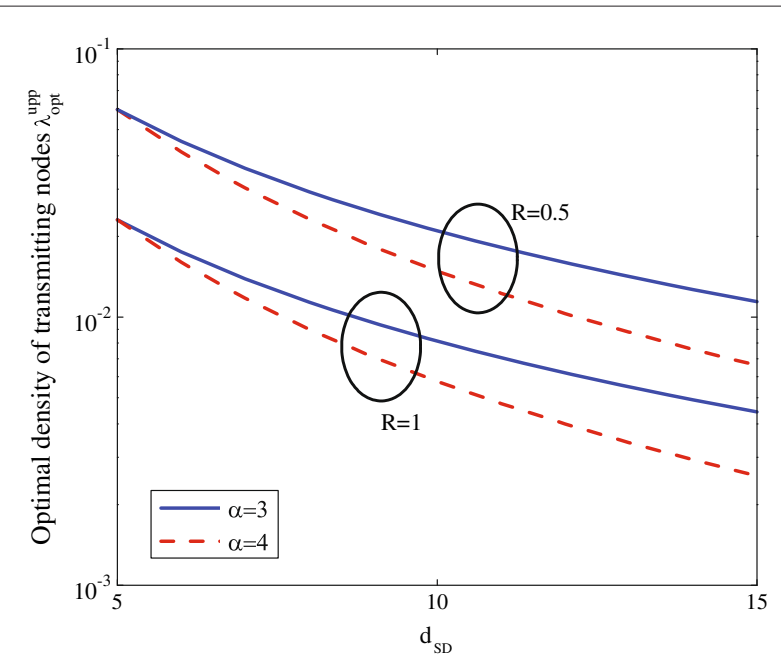

Figure 6 The optimal density of transmitting nodes for multihop AF relaying with $K=5$ as a function of distance between source and destination $\boldsymbol{d}_{S D}$ for different end-to-end spectral efficiency $R=0.5$ and $1 \mathrm{bps} / \mathrm{Hz}$ and path loss exponent $\alpha=3$ and 4 in Rayleigh fading channel with noise variance $\sigma^{2}=1$, transmission power $P_{T}=15 \mathrm{~dB}$, and interference power $P_{I N F}=5 \mathrm{~dB}$.

access transport capacity, but also the optimal spatial density of transmitting nodes decreases due to the attenuation caused by the transmission over a long distance. Furthermore, as expected, the optimal density of transmitting nodes for multihop AF relaying with a low endto-end spectral efficiency and path loss exponent is higher than that with a high spectral efficiency and path loss exponent.

Finally, the upper bound for the optimal density of transmitting nodes as a function of the number of hops $K$ at an end-to-end spectral efficiency $R=0.5 \mathrm{bps} / \mathrm{Hz}$ is plotted in Figure 7 for different end-to-end distances $d_{S D}$ and interference powers $P_{I N F}$, when the transmission power $P_{T}=20 \mathrm{~dB}$ and path loss exponent $\alpha=4$. In this figure, since the Poisson interference accumulates with increasing number of transmission hops in the AF relay networks, we observe that the maximum optimal spatial density is achieved at a specific number of hops $K$. In addition, since the performance degradation caused by attenuation can be the most negative factor, this figure reveals that the effect of interference on the AF relaying is considered relatively small when the multihop transmission over long end-to-end distances.

\section{Conclusion}

This article considered a multihop transmission with AF strategy in the simultaneous presence of both noise and interference, allowing the use of a Poisson interference model. In particular, as all of the nodes are randomly distributed in a wireless ad hoc network, we considered a

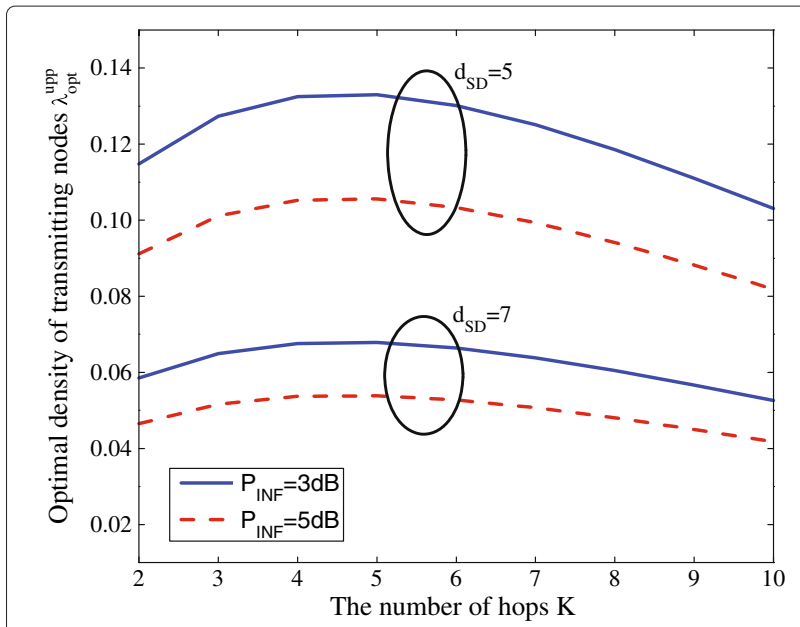

Figure 7 The optimal density of transmitting nodes for multihop AF relaying as a function of number of hops $K$ for different interference power $P_{I N F}$ and distance $d_{S D}$ at end-to-end spectral efficiency $R=0.5 \mathrm{bps} / \mathrm{Hz}$ in Rayleigh fading channel with path loss exponent $\alpha=4$, noise variance $\sigma^{2}=1$, and transmission power $P_{T}=\mathbf{2 0} \mathrm{dB}$.

metric termed random access transport capacity of decentralized wireless networks, to measure the maximum rate of successful end-to-end transmission over some distance in wireless ad hoc networks. Moreover, to compute the random access transport capacity, we derived the exact outage probability of multihop AF relaying, because the performance gap between the conventional bounds and the exact value increases as the number of hops increases. The analytic and simulated results showed that multihop transmission with the AF strategy amplifies the interference as well as the thermal noise and causes an error floor phenomenon in terms of the outage probability. In addition, since there is a tradeoff relationship between the throughput and reliability in terms of the random access transport capacity, we computed the optimal spatial density of transmitting nodes in wireless ad hoc networks. From this article, we can obtain the actual random access transport capacity and predict the maximum number of transmitting nodes per unit area to maximize their performance.

\section{Appendix 1}

\subsection{Proof of end-to-end SINR}

Using our results in [22], the SINR after dual-hop transmission $K=2$ can be written as

$$
\begin{aligned}
\gamma_{\mathrm{e} q} & =\frac{\gamma_{1} \gamma_{2}}{\gamma_{1}+\gamma_{2}+1}=\frac{1}{\frac{1}{\gamma_{1}}+\frac{1}{\gamma_{2}}+\frac{1}{\gamma_{1} \gamma_{2}}} \\
& =\frac{1}{\left(1+\frac{1}{\gamma_{1}}\right)\left(1+\frac{1}{\gamma_{2}}\right)-1}
\end{aligned}
$$


where each $n$th $\operatorname{SINR}$ is given by $\gamma_{n}=\frac{\left|\mathrm{h}_{n}\right|^{2} P_{n}}{\sum_{i \in \Phi_{I_{n}}}\left|\mathrm{~g}_{i}\right|^{2} P_{I_{n}}+\sigma_{n}^{2}}$ for $n=1$ and 2 . Then, the SINR for multihop AF transmission with $K=3$ can be written as

$$
\begin{aligned}
\gamma_{\mathrm{e} q} & =\frac{\left(\frac{\gamma_{1} \gamma_{2}}{\gamma_{1}+\gamma_{2}+1}\right) \gamma_{3}}{\left(\frac{\gamma_{1} \gamma_{2}}{\gamma_{1}+\gamma_{2}+1}\right)+\gamma_{3}+1} \\
& =\frac{\gamma_{1} \gamma_{2} \gamma_{3}}{\gamma_{1}+\gamma_{2}+\gamma_{3}+\gamma_{1} \gamma_{2}+\gamma_{1} \gamma_{3}+\gamma_{2} \gamma_{3}+1} \\
& =\frac{1}{\left(1+\frac{1}{\gamma_{1}}\right)\left(1+\frac{1}{\gamma_{2}}\right)\left(1+\frac{1}{\gamma_{3}}\right)-1} .
\end{aligned}
$$

Expanding the above results, we can obtain the end-toend SINR of multihop AF relaying (7).

\section{Appendix 2}

\subsection{Proof of outage probability of multihop AF relaying}

Using the random variable $X_{n}=\left(1+\frac{1}{\gamma_{n}}\right)$, the end-toend SINR of multihop AF relaying can be rewritten as

$$
\gamma_{\mathrm{e} q}=\frac{1}{\prod_{n=1}^{K}\left(1+\frac{1}{\gamma_{n}}\right)-1}=\frac{1}{\prod_{n=1}^{K} \mathrm{X}_{n}-1} .
$$

Then, we can obtain the $P D F$ of $Z_{K}=\prod_{n=1}^{K} X_{n}$ using the Jacobian of the transformation as

$$
\begin{aligned}
p_{Z_{K}}\left(x_{K}\right)= & \int_{1}^{x_{K}} \frac{1}{x_{K-1}} p_{Z_{K-1}}\left(x_{K-1}\right) p_{\mathrm{x}_{K}} \\
& \left(\frac{x_{K}}{x_{K-1}}\right) d x_{K-1}, \quad \text { for } K \geq 2
\end{aligned}
$$

where

$p_{Z_{K-1}}\left(x_{K-1}\right)=\int_{1}^{x_{K-1}} \frac{1}{x_{K-2}} p_{Z_{K-2}}\left(x_{K-2}\right) p_{\mathrm{X}_{K-1}}\left(\frac{x_{K-1}}{x_{K-2}}\right) d x_{K-2}$.

Using $p_{Z_{1}}\left(x_{1}\right)=p_{\mathrm{X}_{1}}\left(x_{1}\right)$, the $P D F$ of $Z_{K}=\prod_{n=1}^{K} X_{n}$ can be rewritten as

$$
\begin{aligned}
p_{Z_{K}}\left(x_{K}\right)= & \int_{1}^{x_{K}} \cdots \int_{1}^{x_{3}} \int_{1}^{x_{2}}\left(\prod_{n=1}^{K-1} \frac{1}{x_{n}}\right) \\
& {\left[\prod_{n=1}^{K} p_{\mathrm{x}_{n}}\left(\frac{x_{n}}{x_{n-1}}\right)\right] d x_{1} d x_{2} \ldots d x_{K-1} \text { for } K \geq 2 }
\end{aligned}
$$

where $x_{0}=1$.

Finally, using following property

$$
\mathrm{Y}=\frac{1}{\mathrm{X}} \rightarrow p_{\mathrm{Y}}(y)=\frac{1}{y^{2}} p_{\mathrm{X}}\left(\frac{1}{y}\right),
$$

we can obtain the exact outage probability (13) from the following $P D F$ of multihop AF relaying $\gamma_{\mathrm{e} q}=\frac{1}{Z_{K}-1}$ :

$$
p_{\gamma_{\mathrm{e} q}}(s)=\frac{1}{s^{2}} p_{\mathrm{Z}_{K}}\left(1+\frac{1}{s}\right) .
$$

Competing interests

The authors declare that they have no competing interests.

\section{Acknowledgements}

This research was supported by "The Ministry of Knowledge Economy (MKE), Korea, under the Information Technology Research Center (ITRC) support program supervised by the NIPA" (NIPA-2012-H0301-12-3002). The work of S Kim was supported by the Sogang University Research Grant of 2011

\section{Author details}

${ }^{1}$ The School of Electrical Engineering, Korea University, 5-1 Anam-dong, Sungbuk-gu,Seoul, 136-713, Republic of Korea. ${ }^{2}$ The Department of Computer Science and Engineering, Sogang University, Seoul, 121-742, Republic of Korea.

Received: 4 July 2012 Accepted: 7 March 2013

Published: 17 April 2013

\section{References}

1. A Sendonaris, E Erkip, B Aazhang, User cooperation diversity-Part I: system description. IEEE Trans. Commun. 51(11), 1927-1938 (2003)

2. JN Laneman, DNC Tse, GW Wornell, Cooperative diversity in wireless networks: efficient protocols and outage behavior. IEEE Trans. Inf. Theory. 50(12), 3062-3080 (2004)

3. A Bletsas, H Shin, MZ Win, Cooperative communications with outage-optimal opportunistic relaying. IEEE Trans. Wirel. Commun. 6(9), 3450-3460 (2007)

4. B Barua, N HQ, H Shin, On the SEP of cooperative diversity with opportunitic relaying. IEEE Commun. Lett. 12(10), 727-729 (2008)

5. Y Song, $\mathrm{H}$ Shin, EK Hong, MIMO cooperation diversity with scalar-gain amplify-and-forward relaying. IEEE Trans. Commun. 57(7), 1932-1938 (2009)

6. J Boyer, DD Falconer, $\mathrm{H}$ Yanikomeroglu, Multihop diversity in wireless relaying channels. IEEE Trans. Commun. 52(10), 1820-1830 (2004)

7. GK Karagiannidis, TA Tsifitsis, RK Mallik, Bounds for multihop relayed communications in Nakagami-m Fading. IEEE Trans. Commun. 54, 18-22 (2006)

8. G Farhadi, NC Beaulieu, On the ergodic capacity of multi-hop wireless relaying systems. IEEE Trans. Wirel. Commun. 8(5), 2286-2291 (2009)

9. G Farhadi, NC Beaulieu, Capacity of amplify-and-forward multi-hop relaying systems under adaptive transmission. IEEE Trans. Commun. 58(3), 758-763 (2010)

10. HQ Ngo, EG Larrson, Linear multihop amplify-and-forward relay channels: error exponent and optimal number of hops. IEEE Trans. Wirel. Commun. 10(11), 3824-2842 (2011)

11. I Krikidis, SMJS Thompson, N Geortz, Max-min relay selection for legacy amplify-and-forward systems with interference. IEEE Trans. Wirel. Commun. 8(6), 3016-3027 (2009)

12. C Zhong, S Jin, KK Wong, Dual-hop system with noisy relay, and interference-limited destination. IEEE Trans. Commun. 58(3), 764-768 (2010)

13. T Soithong, VA Aalo, GP Efthymoglou, C Chayawan, Outage analysis of multihop relay systems in interference-limited Nakagami- $m$ fading channels. IEEE Trans. Veh. Technol. 61(3), 1451-1477 (2012)

14. SS Ikki, S Aissa, Multihop wireless relaying systems in the presence of cochannel interference: performance analysis and design optimization. IEEE Trans. Veh. Technol. 61(2), 566-573 (2012)

15. SP Weber, X Yang, JG Andrews, G de Veciana, Transmission capacity of wireless ad hoc networks with outage constraints. IEEE Trans. Inf. Theory. 51(12), 4091-4102 (2005)

16. S Weber, JG Andrews, N Jindal, The effect of fading, channel inversion, and threshold scheduling on ad hoc networks. IEEE Trans. Inf. Theory. 53(11), 4127-4149 (2007)

17. AM Hunter, JG Andrews, S Weber, Capacity scaling of ad hoc networks with spatial diversity. IEEE Trans. Wirel. Commun. 7(12), 5058-5071 (2008)

18. S Weber, JG Andrews, X Yang, G de Veciana, Transmission capacity of wireless ad hoc networks with successive interference cancellation. IEEE Trans. Inf. Theory. 53(8), 2799-2814 (2007)

19. S Weber, JG Andrews, $N$ Jindal, An overview of the transmission capacity of wireless networks. IEEE Trans. Commun. 58(12), 3593-3604 (2010)

20. V Chandrasekhar, JG Andrews, Spectrum allocation in tiered cellular networks. IEEE Trans. Commun. 57(10), 5314-5327 (2009)

21. R Vaze, Throughput-Delay-Reliability tradeoffs with $A R Q$ in wireless ad hoc networks. IEEE Trans. Wirel. Commun. 10(7), 2142-2149 (2011) 
22. J Lee, H Shin, J Heo, Transmission capacity for dual-hop relaying in wireless ad hoc networks. EURASIP J. Wirel. Commun. Network. 1(58), 1-10 (2012)

23. JG Andrews, S Weber, M Kountouris, M Haenggi, Random access transport capacity. IEEE Trans. Wirel. Commun. 9(6), 2101-2111 (2010)

24. D Daley, D Vere-Jones. (Springer, Berlin, 2003), pp. 19-40

25. M Haenggi, RK Ganti, Interference in Large Wireless Networks now-The essence of knowledge. Massachusetts, 147-185 (2010)

26. J J Lee, H Shin, J Heo, in IEEE Wireless Communications and Networking Conference (WCNC'12). Random access transport capacity of dual-hop AF relaying in a wireless ad hoc networks (France, Paris, 2012)

doi:10.1186/1687-1499-2013-104

Cite this article as: Lee et al:: Random access transport capacity of multihop AF relaying: a throughput-reliability tradeoff. EURASIP Journal on Wireless Communications and Networking 2013 2013:104.

Submit your manuscript to a SpringerOpen ${ }^{\circ}$ journal and benefit from:

- Convenient online submission

Rigorous peer review

- Immediate publication on acceptance

- Open access: articles freely available online

- High visibility within the field

- Retaining the copyright to your article

Submit your next manuscript at $\boldsymbol{\triangleright}$ springeropen.com 